\title{
KRAS and BRAF Concomitant Mutations in a Patient with Metastatic Colon Adenocarcinoma: An Interesting Case Report
}

\author{
Concetta Cafiero $^{a} \quad$ Agnese Re $^{b} \quad$ Gerardo D'Amato $^{c}$ Pier Luigi Surico ${ }^{d}$ \\ Giammarco Surico $^{d}$ Michele Pirrellie $^{e}$ Salvatore Pisconti ${ }^{a}$ \\ aUnità di Oncologia Medica, Ospedale S.G. Moscati, Taranto, Italy; 'bstituto di Analisi \\ dei Sistemi ed Informatica "Antonio Ruberti" (IASI), Consiglio Nazionale delle Ricerche \\ (CNR), Rome, Italy; 'Dipartimento di Chirurgia, Fondazione Policlinico A. Gemelli, Rome, \\ Italy; 'Unità di Oncologia, Ente Ecclesiastico Miulli, Acquaviva delle Fonti, Italy; eUnità di \\ Anatomia Patologica, Ospedale SS Annunziata, Taranto, Italy
}

\section{Keywords}

Colorectal cancer $\cdot$ KRAS $\cdot B R A F \cdot$ Therapeutic responses

\begin{abstract}
A 68-year-old female patient with tenesmus and blood in the stool was admitted to the S.G. Moscati Hospital of Taranto. Investigations revealed infiltrative mucinous colon adenocarcinoma accompanied by lymph node metastases. Following surgery and adjuvant chemotherapy, computed tomography $(\mathrm{CT})$ and carcinoembryonic antigen screening were negative. Two years later, CT demonstrated a liver lesion. Histologic and genetic analyses confirmed the diagnosis of metastatic colorectal cancer with the coexistence of KRAS and BRAF mutations in hepatic metastases and the presence of the BRAF V600E in the primary tumour. It is unclear whether the lack of response was due to $B R A F$ mutations, but the data suggest that mutated $B R A F$ confers resistance to anti-epidermal growth factor receptor therapy. In our patient, $B R A F$ mutation turned out to be a negative prognostic factor, and it may have been the cause of clinical implications for disease progression and therapeutic responses.
\end{abstract}

(C) 2020 The Author(s).

Published by S. Karger AG, Basel

\section{Introduction}

Colorectal cancer (CRC) is the third most commonly diagnosed cancer and cause of death among men and women. At stage IV of CRC, liver metastases occur in $20-30 \%$ of patients, whereas peritoneal and lung metastases occur in $10-15 \%$ and $10-25 \%$ of patients, respectively, and other non-rectal or non-colon metastases occur rarely [1]. CRC progresses through 
a series of well-defined steps associated with specific genetic and epigenetic alterations in various oncogenes and tumour suppressor genes [2]. Approximately 50-60\% of colorectal tumours are mutated (missense mutation) in the KRAS gene, and approximately $5-10 \%$ of tumours show mutation in the BRAF gene (missense mutation). These mutations in KRAS and BRAF oncoproteins activate signalling cascades that mediate cellular responses such as cell proliferation, apoptosis, adhesion, invasion and angiogenesis. Mutations in RAS genes (KRAS exons 2, 3 and 4 and $N R A S$ exons 2, 3 and 4) located downstream from epidermal growth factor receptor (EGFR) within this pathway lead to its activation even if EGFR is blocked [2, 3]. Although the BRAF gene is located downstream of $K R A S$, the activating V600E BRAF mutation is not considered a predictive biomarker for resistance to anti-EGFR antibody therapy. However, mutations in this gene have been suggested to be strong prognostic markers of poor prognosis in CRC patients [4].

The EGFR signalling pathway becomes constitutively active in these tumours, so that now the strategy of drug development is moving towards skilled aiming at the RAS pathway [5]. Molecular screening, such as for Val600Lys in the BRAF gene, is crucial for the care of CRC patients, and it might significantly improve the cost-effectiveness and important consequences regarding treatment. In this study, we report a case of metastatic CRC with coexistent $K R A S$ and $B R A F$ mutations in a 68-year-old woman affected by advanced adenocarcinoma of the rectum and liver metastases. Concomitant KRAS and BRAF mutation in CRCs is rare, occurring in less than $0.001 \%$ of cases [6], but this event appears to be associated with the presence of $B R A F$ mutation in the primary tumour and with a more aggressive outcome, as in this case. These are two activators in the protooncogenes that induce a functional loss of tumour suppressor genes. RAF mutations in CRC are mostly V600E amino acid substitutions, although various other mutations at codon 600 or neighbouring positions within the kinase domain are documented, too. Structural studies of RAF proteins have identified the valine at position 600 as a crucial site within the conserved kinase domain, which is required for BRAF to maintain an inactive conformation in the absence of KRAS-BRAF interaction [7]. Mechanistically, mutations at this site likely render BRAF constitutively active, bypassing dimerization with BRAF or RAF1, which is normally a prerequisite for activation. Consequently, the V600E mutation is strongly activating, resulting in constitutive MEK binding, phosphorylation and therefore BRAF signal transduction. BRAF amplification and/or loss of heterozygosity have infrequently been detected in CRC [8]. The significance of these $B R A F$ genomic imbalances is unclear; however, $B R A F$ copy number gains have been implicated in drug resistance of CRC. Metastatic CRC with concomitant $R A S+B R A F$ mutations should be assigned to a separate arm in clinical trials to evaluate the role of novel therapeutics for this deadly disease.

\section{Case Presentation}

\section{Patient and Treatments}

In July 2015, a 68-year-old female patient with rectal tenesmus and blood in the stool underwent colonoscopy at the S.G. Moscati Hospital of Taranto. The examination revealed a fungating and bleeding stenotic mass. Histologic analysis of a biopsy from this mass supported the diagnosis of adenocarcinoma. A total body computed tomography (CT) scan showed a thickening of the descending colon wall and the presence of pericentromeric lymph nodes in the pericolic fat tissue. After a few days, the patient was admitted to the Surgery Department, SS Annunziata Hospital of Taranto, and colectomy and splenectomy were subsequently carried out. 
The final histologic diagnosis was infiltrative mucinous adenocarcinoma, with metastases in 4 out of 17 resected lymph nodes, but no pathological aspects were observed in the spleen (pT3pN2aMx G2). After surgery, the patient received adjuvant chemotherapy with 12 cycles of FOLFOX regimen (fluorouracil + folinic acid + oxaliplatin). Postoperative CT scan examination was negative, and carcinoembryonic antigen and gastrointestinal cancer antigen levels were within the normal range. In February 2017, a total body CT scan evidenced a suspicious liver lesion between segments VI and VII and a liver biopsy was carried out. Histologic analysis confirmed the colic origin of the metastasis by positivity for CK20 and CDX2. Metronomic treatment with capecitabine was started.

After obtaining informed consent from the patient, the immunohistochemical EGFR expression profile was investigated by anti-EGFR monoclonal antibody according to the manufacturer's descriptions. Paraffin-embedded tissue sections were collected on microscopic slides. Molecular assessment of the KRAS and BRAF genes on the liver biopsy and the primary tumour was performed at the Pathology Department, SS Annunziata Hospital of Taranto. Haematoxylin and eosin slides were reviewed by the pathologist to confirm the diagnosis and select the best representative area of the tumour for DNA extraction.

\section{DNA Extraction}

Tree 10- $\mu$ m-thick unstained sections were cut from the previously selected paraffin blocks. The slides were deparaffinized with xylene. The neoplastic tissue sample was obtained by manual macrodissection. DNA was extracted with a QIAcube Instrument (QIAGEN). The DNA concentration was measured on a QUBIT instrument (Thermo Fisher Scientific, Waltham, MA, USA), and the minimum DNA concentration for the experiments was set to $2 \mathrm{ng} / \mu \mathrm{L}$.

\section{Analysis of KRAS and BRAF Mutations}

Mutation analysis was conducted by pyrosequencing in the coding sequence of the KRAS gene (exon 2 and exon 3 ) and the BRAF gene (exon 15) using the therascreen ${ }^{\circledR} K R A S$ and $B R A F$ Pyro Kits, respectively.

\section{Results}

In hepatic tissue, the identified alterations in the KRAS and $B R A F$ genes were a mutation in codon 12 (c.35G>A p.G12D) of exon 2 and a missense nucleotide base change in codon 600 (c.1799T $>$ A GTG to GAG p.V600E) of exon 15, respectively, while in the primary tumor there was only BRAF mutation.

The patient's case was discussed by a multidisciplinary tumour board. The board's recommendation was for upfront systemic treatment with 12 cycles of FOLFIRI (5FU) + Avastin (bevacizumab) and a next evaluation pending determination of a response to chemotherapy (January 2018). A total body CT in July 2018 showed pulmonary microlesions, increased hepatic lesions and deep venous thrombosis of the left gonadal vein. The patient's case was discussed by the multidisciplinary tumour board, and in August the patient received chemotherapy with Stivarga (regorafenib) $40 \mathrm{mg}$, starting with 2 tablets/day for 1 week and then 3 tablets/day for 3 weeks. The course of treatment was complicated by side effects: asthenia, lack of appetite and resistant shoulder pain. A total body CT in November 2018 showed increased pulmonary, hepatic and renal lesions. In December 2018, the patient received third-line therapy with FOLFOX at low doses and oral Lonsurf (trifluridine/tipiracil). After 3 weeks, clinical improvement was reported with lack of appetite and good pain control. A total body CT in July 2019 showed only increased pulmonary lesions. 
Cafiero et al.: KRAS and BRAF Metastatic Colon Adenocarcinoma

\section{Discussion}

This rare case of metastatic CRC with coexistent KRAS and BRAF mutations had several unusual features, including rapid progression of disease. It has been shown that patients who have both KRAS and BRAF mutations tend to have an adverse outcome [9-12]. No possible mechanism underlying coexistent $K R A S$ and $B R A F$ mutation is known. Furthermore, it is unclear whether these tumours have a different biology and natural history than single KRAS or BRAF mutant tumours, or which of the two mutations is the dominant oncogene driving tumour proliferation [10-14]. It is known that most $B R A F$ mutations identified in CRC are V600E, which is a class I mutation. The valine at codon 600 lies within the kinase domain and is required for BRAF to maintain an inactive status in the absence of KRASBRAF interaction. The V600E mutation results in amino acid substitution from a valine to a glutamic acid, leading to 130 - to 700 -fold increased BRAF kinase activity compared with wild-type $B R A F$ [15]. In metastatic CRC, patients with BRAF V600E mutation are not likely responding to anti-EGFR therapy, and they have decreased survival compared to patients with wild-type BRAF [16]. Jones et al. [15] have reported that non-V600E BRAF mutant metastatic CRC represents a clinically distinct molecular subtype, associated with significantly longer overall survival compared to that of metastatic CRC patients with a BRAF V600E mutation. A possible mechanism to be considered is the presence of a biclonal population of cancer cells, with a clone harbouring a KRAS mutation and the other clone harbouring a $B R A F$ mutation.

The impact of $B R A F$ mutations has also been retrospectively evaluated on tissue from completed prospective trials. MRC (Medical Research Council) COIN was the largest trial that studied the effect of the addition of anti-EGFR treatment (cetuximab) to a chemotherapy regimen of fluoropyrimidine in metastatic CRC [17]. The effect of cetuximab was further analysed for the presence/absence of KRAS, NRAS and BRAF mutations. The data showed that the addition of anti-EGFR drugs to standard chemotherapy for $B R A F$ mutant metastatic CRC is associated with worse outcomes. In fact, the median overall survival was shorter with $B R A F$ mutant CRC (8.8 months) than with $B R A F$ and $K R A S$ wild-type tumours (17.5 months) [11-14]. Bevacizumab is a drug approved by the FDA and used as an inhibitor of $B R A F \mathrm{~V} 600 \mathrm{E}$ in $B R A F$ mutant melanoma. It has also been tested in $B R A F$ mutant CRC but failed to show any clinical advantage and antitumour activity [18]. When bevacizumab blocks $B R A F$ activity and cuts off signalling within the MAPK pathway, this kicks on a feedback mechanism leading to upregulation of upstream EGFR, once again driving signalling through the MAPK pathway upon which these tumours are so dependent. Thus, the optimal management of metastatic CRC harbouring concomitant KRAS and BRAF mutation is still unknown.

\section{Conclusion}

In standard clinical management of CRC, KRAS mutation serves as a predictive biomarker for the selection of patients eligible for anti-EGFR therapy, with a benefit recorded only for $R A S$ wild-type tumours. As reported, concomitant $K R A S$ and $B R A F$ mutation is associated with more severe disease, and this emphasizes the importance of obtaining baseline testing of these mutations as a standard of care in the clinical management of metastatic CRC patients. Furthermore, this distinct and highly aggressive subset of tumours should be assigned to a separate arm in clinical trials to evaluate novel therapeutic approaches. 


\section{Statement of Ethics}

All procedures were conducted according to the principles expressed in the Declaration of Helsinki and the Guideline for Good Clinical Practice. The patient provided written informed consent to participate, as well as for the publication of any relevant clinical information for scientific purposes.

\section{Disclosure Statement}

The authors have no conflicts of interest to declare.

\section{Funding Sources}

This research did not receive any financial support from funding agencies in the public, commercial or not-for-profit sectors.

\section{Author Contributions}

S.P. participated in the clinical diagnosis and the management of the patient. M.P. performed the histologic analysis. G.S. participated in the management of the patient. P.L.S. participated in the clinical diagnosis. C.C. performed the molecular genetic study and variant identification and drafted the manuscript. A.R. participated in molecular analysis and in the drafting of the manuscript. G.D. revised the work. All authors contributed to critical discussion and approved the final version of the manuscript.

\section{Availability of Data and Materials}

DNA from the patient and the original pyrosequencing are available upon request.

\section{References}

1 Cirocchi R, Trastulli S, Abraha I, Vettoretto N, Boselli C, Montedori A, et al. Non-resection versus resection for an asymptomatic primary tumour in patients with unresectable stage IV colorectal cancer. Cochrane Database Syst Rev. 2012 Aug;(8):CD008997.

2 Cancer Genome Atlas Network. Comprehensive molecular characterization of human colon and rectal cancer. Nature. 2012 Jul;487(7407):330-7.

3 Di Nicolantonio F, Martini M, Molinari F, Sartore-Bianchi A, Arena S, Saletti P, et al. Wild-type BRAF is required for response to panitumumab or cetuximab in metastatic colorectal cancer.J Clin Oncol. 2008 Dec;26(35):570512.

4 Morikawa T, Inada R, Nagasaka T, Mori Y, Kishimoto H, Kawai T, et al. BRAF V600E mutation is a predictive indicator of upfront chemotherapy for stage IV colorectal cancer. Oncol Lett. 2018 Feb;15(2):2195-201.

5 Bokemeyer C, Van Cutsem E, Rougier P, Ciardiello F, Heeger S, Schlichting M, et al. Addition of cetuximab to chemotherapy as first-line treatment for KRAS wild-type metastatic colorectal cancer: pooled analysis of the CRYSTAL and OPUS randomised clinical trials. Eur J Cancer. 2012 Jul;48(10):1466-75.

6 Sahin IH, Kazmi SM, Yorio JT, Bhadkamkar NA, Kee BK, Garrett CR. Rare though not mutually exclusive: a report of three cases of concomitant $K R A S$ and $B R A F$ mutation and a review of the literature. J Cancer. 2013; 4(4):320-2.

7 Gallo G, Sena G, Vescio G, Papandrea M, Sacco R, Trompetto M, et al. The prognostic value of KRAS and BRAF in stage I-III colorectal cancer. A systematic review. Ann Ital Chir. 2019;90:127-37. 
8 Wan PT, Garnett MJ, Roe SM, Lee S, Niculescu-Duvaz D, Good VM, et al. Mechanism of activation of the RAF-ERK signaling pathway by oncogenic mutations of B-RAF. Cell. 2004 Mar;116(6):855-67.

9 Ma YS, Huang T, Zhong XM, Zhang HW, Cong XL, Xu H, et al. Correction to: Proteogenomic characterization and comprehensive integrative genomic analysis of human colorectal cancer liver metastasis. Mol Cancer. 2019 Apr;18(1):72.

10 Vittal A, Middinti A, Kasi Loknath Kumar A. Are all mutations the same? A rare case report of coexisting mutually exclusive $K R A S$ and $B R A F$ mutations in a patient with metastatic colon adenocarcinoma. Case Rep Oncol Med. 2017;2017:2321052.

11 Barton S, Starling N, Swanton C. Predictive molecular markers of response to epidermal growth factor receptor (EGFR) family-targeted therapies. Curr Cancer Drug Targets. 2010 Dec;10(8):799-812.

12 Cree IA, Deans Z, Ligtenberg MJ, Normanno N, Edsjö A, Rouleau E, et al. Guidance for laboratories performing molecular pathology for cancer patients. J Clin Pathol. 2014 Nov;67(11):923-31.

13 Douillard JY, Siena S, Cassidy J, Tabernero J, Burkes R, Barugel M, et al. Final results from PRIME: randomized phase III study of panitumumab with FOLFOX4 for first-line treatment of metastatic colorectal cancer. Ann Oncol. 2014 Jul;25(7):1346-55.

14 Maughan TS, Adams RA, Smith CG, Meade AM, Seymour MT, Wilson RH, et al. Addition of cetuximab to oxaliplatin-based first-line combination chemotherapy for treatment of advanced colorectal cancer: results of the randomised phase 3 MRC COIN trial. Lancet. 2011 Jun;377(9783):2103-14.

15 Jones JC, Renfro LA, Al-Shamsi HO, Schrock AB, Rankin A, Zhang BY, et al. BRAF mutations define a clinically distinct molecular subtype of metastatic colorectal cancer. J Clin Oncol. 2017 Aug;35(23):2624-30.

16 Modest D, Camaj P, Heinemann V, Schwarz B, Jung A, Laubender R, et al. KRAS allel-specific activity of sunitinib in an isogenic disease model of colorectal cancer. J Cancer Res Clin Oncol. 2013 Jun;139(6):953-61.

17 Van Cutsem E, Köhne CH, Láng I, Folprecht G, Nowacki MP, Cascinu S, et al. Cetuximab plus irinotecan, fluorouracil, and leucovorin as first-line treatment for metastatic colorectal cancer: updated analysis of overall survival according to tumor KRAS and BRAF mutation status. J Clin Oncol. 2011 May;29(15):2011-9.

18 Davies H, Bignell GR, Cox C, Stephens P, Edkins S, Clegg S, et al. Mutations of the BRAF gene in human cancer. Nature. 2002 Jun;417(6892):949-54. 